\title{
Improving Quality of Service (QoS) in Mobile Information Systems, Services and Networks
}

In this special double issue, we present eight selected papers presented at the Fourth International Conference on Advances in Mobile Computing \& Multimedia (MoMM2006), held in Yogyakarta, Indonesia from the $4^{\text {th }}$ to the $6^{\text {th }}$ of December 2006.

These papers highlight the need for improving Quality of Service $(\mathrm{QoS})$ in mobile applications. The improvement can be achieved by having frameworks and measurement criteria perceived by the users, as described by the first two papers. The improvement can be achieved by increasing Internet Protocol technologies, as they are shown by the third and the fourth papers. Finally, the improvement can also be met by having higher performance, dependable and more predictable networks as is presented by the next four papers.

The first paper by Bohl, Manouchehri and Winand presents the current emphasis of using mobile information systems in our everyday life. Not only business environments, but also private households now demand the use of mobile services in daily basis. Unfortunately, unlike in business environments, the mobile services have not been fully developed for the private sector. In this paper, the authors propose a methodical framework that combines the mobile processes characteristics in private everyday life with the current support of mobility offered by mobile applications, devices and infrastructures. The framework is explained using several case studies. The initial stage of evaluation plan is also presented. Having a combination of available technologies and users' perspective can improve the QoS of the mobile information system framework.

In the second paper, Agboma and Liotta present the research on how users perceive mobile content delivery services. The content relates to multimedia services such as television programs and videos on mobile terminals such as 3G Mobile Phone, Personal Digital Assistant (PDA) and Laptop. The paper claims that the QoS management should be driven by the user perception of quality, indicated by the users Quality of Experience (QoE). This is opposed to the traditional QoS measurement that is usually described by raw engineering parameters such as latency, jitter, bandwidth and so on.

In the next paper, Gehlen, Aijaz, Zhu and Walke propose a framework that supports Mobile P2P (peerto-peer) Web Services. Mobile Web Services are usually known to have bad QoS due to the frequent mobile network change. In this paper, the authors resolve the issue by using Session Initiation Protol (SIP) in their framework. For proof of concept, an exemplary mobile chess game in J2ME devices has been used. The performance in terms of response time using this framework has been improved.

IP Multimedia Subsystem (IMS) is a network framework based on the internet protocols that allows service providers to deliver services to end users independently of their device type, network type and physical location. IMS has the ability to provide a number of generic fundamental functions that can 
be reused by services. In the next paper, Lin and Liotta, emphasize one of the services, the presence service. This service enables a user to communicate with other users. The authors build a prototype to study the performance of IMS presence service. Based on the study, they find that the presence service specified by IMS standard does not perform well in terms of scalability.

In the next paper, Saqour, Shanuldin and Ismail study the inaccuracy location information caused by mobility metrics (bacon interval and nodel speed) on geographical GPSR (Greedy Perimeter Stateless Routing) both in mobile and ad-hoc network protocol. This problem is called Neighbor Break Link (NBL). The paper proposes various prediction schemes to overcome these problems.

In the sixth paper, Simon and Imre present an algorithm to minimize signalling overhead cost in mobile networks. This cost is incurred by the high volume of location management operations and call deliveries. For algorithm simulation, the authors use a realistic mobile environment simulator to generate input. The result shows that their proposed algorithm can reasonably reduce the amount of signalling traffic and thus increase the mobile networks QoS.

In the next paper, Ishak, Xu, Olariu and Salleh highlight the locationing problem in Wireless Sensor Networks(WSNs). This problem can be viewed as how sensors can discover nodes and estimate ranges between nodes. The authors argue that to achieve this, the sensors require coarse-grain location awareness. They propose a hybrid training protocol to train the sensors in learning their coarse-grain location. The protocol is then evaluated using a set of simulation.

The last paper, by De Marco, Yang, Ikeda, and Barolli, presents a performance evaluation of applications running on top of WSN. The authors perform simulation of WSN with a single sink and a number of sensor nodes feeders, which detect all irregularities in real life environments. The paper shows, via simulation, how event-reliability can be maintained by detecting the presence of irregularities in radio channel.

The guest editor would like to thank IOS Press, MOBIS Editor-in-Chief and ad-hoc reviewers for the collaboration opportunity in this special issue. Finally, the guest editor would like to thank the authors who have prepared their papers and revised them in accordance to the reviewers' feedbacks in a timely manner.

Eric Pardede is a lecturer in the Department of Computer Science and Computer Engineering, La Trobe University, Australia. $\mathrm{He}$ received his $\mathrm{PhD}$ in Computer Science in 2006 from the same institution. His current research interests include XML and Web data management, mobile database, data quality and data integration. He has published around 30 papers in journals, books and various international conferences and workshops. He has co-authored a book entitled Object-Oriented Oracle.

Dr. Pardede is serving as program chair, publicity chair and organizer of several international conferences and workshops. $\mathrm{He}$ is also serving as a program committee member and reviewer for various conference and journals. 

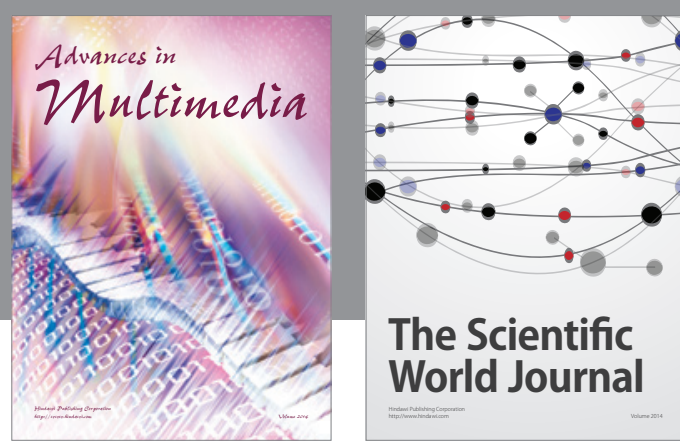

The Scientific World Journal
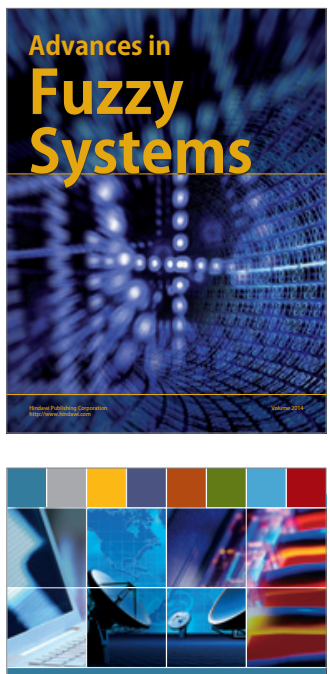

Computer Networks and Communications
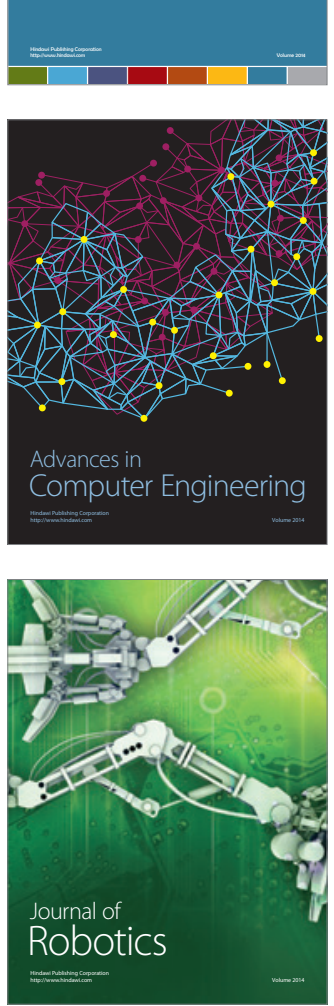
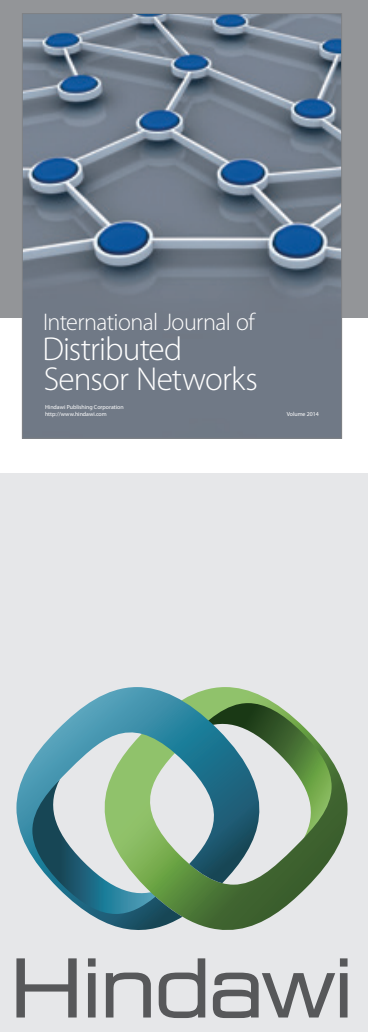

Submit your manuscripts at

http://www.hindawi.com
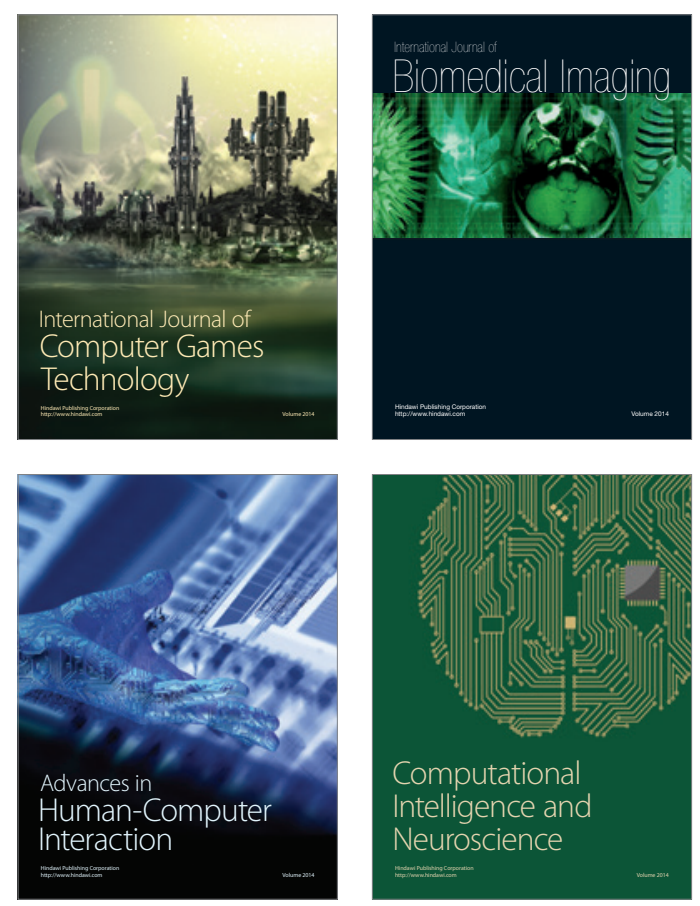
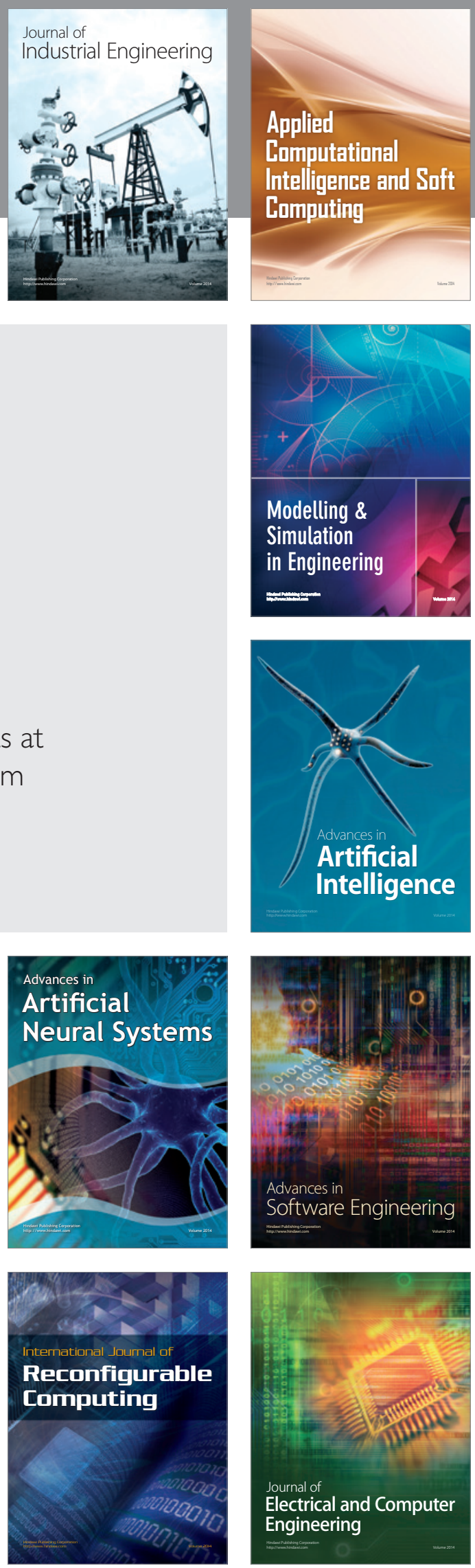